\title{
ALECSO mobile apps initiative
}

\author{
Mohamed Jemni* ${ }^{*}$ and Mohamed Koutheair Khribi
}

\begin{abstract}
Technological advancements in wireless and broadband networks infrastructures have promoted the widespread distribution of mobile devices. Accordingly, content and software developers rushed to adapt their products, and to innovate and create appropriate services and applications to be used in the everyday life of mobile devices' users. Recent stats from the International Telecommunication Union (ITU) unveiled that mobile broadband is the fastest-growing ICT service in history, and around 4 billion of the global mobile phone population have Internet access using their mobile devices. In light of these considerations, there is no doubt that mobile technologies are entirely changing our lives, including particularly the way we learn. Nevertheless, when trying to draw up the status of Arab mobile Apps development and use, several difficulties show up, mainly in relation to the very few available Arab mobile applications in international apps stores. Therefore, recognizing the potentials of mobile technologies and its status in the region, the Arab League Educational, Cultural, and Scientific Organization (ALECSO), has proposed a Pan-Arab wide mobile initiative, aiming to promote the use and development of mobile applications in the Arab world, particularly in the fields of education, culture, science, and serious games. In this paper, we present an overview of the ALECSO mobile initiative, and we describe in details its related activities and implementations.
\end{abstract}

Keywords: Mobile Apps, Mobile Markets, Mobile learning, Cross-Platform Development, Smart Learning

\section{Background}

Recent years have witnessed an increasing interest in the mobile computing realm. Gartner says that mobile device (smartphones and tablets) sales are on a continuous rise from year to year, and the future of enterprise applications, both for enterprise workers and consumers, is mobility. According to ITU ${ }^{1}$ stats, more than $90 \%$ of the global population are covered by a mobile-cellular signal, and more than the half of the population of the Earth are using now mobile communications. This means that mobile devices, to which access has become ubiquitous, are extremely influencing national economies worldwide, and thus empowering people and society, especially in the areas of job creation, entrepreneurship and economic growth in both developed and developing regions (GSM Association 2015a). In the Mobile Economy report authored by GSMA, ${ }^{2}$ it was stated that: "There are still many adults and young people who would appreciate the social and economic benefits of mobile

\footnotetext{
* Correspondence: mohamed.jemni@alecso.org.tn

ICT Department Arab League Educational, Cultural and Scientific

Organization ALECSO, Av. Med Ali Akid, Centre Urbain Nord, BP11201003

Tunis, Tunisia
}

technology but are unable to access it, highlighting a huge opportunity for future growth and a challenge to all players in the industry ecosystem to expand the scope of products and services to tap this demand." (Association GSM and ATKEARNEY 2013). This statement refers to the ongoing need for mobile services, while plenty of opportunities remain unexploited, but can be seized thanks to the large scope of mobile technologies and resulting positive impacts on society and economy. So what is the status of mobile technologies in Arab countries? Are there sufficiently suitable Arab Mobile Apps? Is there a global mobile Arab market suitable for gathering and promoting these apps?

Actually, all of these questions were raised in the Arab League Educational, Cultural and Scientific Organization (ALECSO) precisely, during the preparation of its forthcoming ICT projects for the two-year financial plan 2015-2016. Within this context, ALECSO launched an internal study to assess the status of Mobile technologies in Arab countries. This study showed that the mobile industry has grown rapidly over the last few years in the Arab world. Official stats (GSM Association 2015b) revealed that $54 \%$ of the population is subscribed to a 
mobile service as of mid-2015. In 2014, mobile technologies and services generated 4\% of GDP in the Arab States, a contribution that amounted to around $\$ 115$ billion of economic value across 18 Arab countries. Naturally, with such rapid increase in mobile devices adoption, apps have become increasingly more popular. Indeed, around half of users in Egypt, Lebanon, Saudi Arabia and the UAE downloaded more than five apps (both paid and free) in June 2015, the most popular application types are tied to gaming and social media. It is worth noting that most customers who paid for apps have also made in-app purchases (G. Business 2015). Then, what's about the remaining half? In fact, the main reasons, preventing the remaining half of mobile devices' users in these countries (and mostly in all Arab countries) from using and purchasing apps, are the following:

- Lack of the required and suitable apps;

- Very few number of appropriate available applications in Arabic language existing so far;

- No availability of online payment using credit cards and foreign currencies in international mobile markets in several Arab countries. (Actually, the latter reason is even prohibiting some Arab Mobile developers to submit their apps and to get profits)

Knowing also that mobile apps and content continue to be dominated by a few major languages, most significantly English. Whereas Arabic language, as well as a significant number of other national languages, is only represented by less than $0.1 \%$ over the Internet. Moreover, according to ITU, future first-time Internet and mobile users are likely to come mainly from less wellrepresented languages and dialects, less well-educated, and less urban backgrounds.

For all of these aforementioned considerations, ALECSO has proposed the Arab mobile Apps initiative and has accordingly launched a set of promising related projects aiming to support the Arab Mobile Apps use and development, especially, in relation to the areas of education, culture and science in the Arab region (Jemni 2014). The overall goal of this initiative is to promote the Arab mobile industry and to prepare the Arab digital ecosystem for the new mobile technology opportunities, harnessing region's assets (common language, global market, youth population, decent mobile use penetration, etc.) and mobile benefits (economic growth and entrepreneurship, and major improvements in education, financial, healthcare, social, services and other priority sectors) (ALECSO and ITU 2015). This paper is arranged in the following way: In section 2 , we evoke the main existing initiatives and works related to Mobile technologies promotion. In section 3, we describe the ALECSO Mobile Apps initiative. In section 4, we focus on the ALECSO Apps initiative projects and activities, namely, the ALECSO Apps Store, Editor, Award and training projects. In section 5, we present an ongoing multimodal training program called ALECSO M-Developer Program aiming to build and reinforce capacities in the region in the field of mobile applications' development harnessing online learning facilities. In section 6, we present first exploration of the impact of the initiative in the region and to which extent people in Arab countries react and adhere to its underlying projects and activities. Finally, a conclusion is presented.

\section{Mobile initiatives}

In the last decade, several projects regarding the use of mobile technology especially in education have been carried out all over the world. The primary motives are the widespread use of mobile devices thanks to their low cost and their multiple and diverse functionalities. There has been also a growing recent interest in experimenting with mobile devices especially tablets in schools adopting BYOD (Bring Your Own Device), BYOT (Bring Your Own Technology), and mobile learning approaches. Many pilot projects have been conducted in this context whether through personal initiatives driven by enthusiastic leaders (e.g. teachers) at small scales or within regional/national initiatives and/or policies to promote mobile learning and ICT use in education. These initiatives aim generally to promote the use of mobile technologies whether at an educational level and/or at a larger scale. However, all of these practices are still emergent, and differ from one country to another.

In fact, mobile learning involves the use of mobile technology, either alone or in combination with other information and communication technology (ICT), in order to enable learning anytime and anywhere. Learning can unfold in a variety of ways: people can use mobile devices to access educational resources, connect with others, or create content, both inside and outside classrooms. Mobile learning also encompasses efforts to support broad educational goals such as the effective administration of school systems and improved communication between schools and families

In the European union for instance, the European Commission has funded a number of successful projects related to mobile technologies and smart learning, mostly dedicated to develop a primarily technological infrastructure with respect to the learner-centered approach, in which mobile technologies play a part, alongside more fixed technologies, in supporting ubiquitous learning (The United Nations Educational Scientific and Cultural Organization UNESCO 2012a). In (KukulskaHulme et al. 2011), Kukulska-Hulme et al. describe the most important EU-funded R\&D projects over the past decade. MOBIlearn is a mobile learning initiative that 
ran from 2002 to 2005 with funding from the European Commission involving nine European countries as well as several countries outside of Europe. Universities and mobile phone and telecommunications companies are the main actors in this programme. The main objective is to enhance learning using mobile technologies, which would support and develop learning outside of traditional schools and classrooms. The eMapps programme is another mobile initiative that ran from 2005 to 2008 with support of the European Commission. The eMapps project aims to develop the skills of creativity and innovation among communities of technologically-savvy and internationally-minded children who would generate digital content about their local culture and exchange experiences and communicate with peers in other countries through eMapps' platform and network. The project aims also to build adaptable interactive tools, mainly serious games played on a mobile platform, in order to thrive children' abilities for analysis, interpretation, problem solving, memory exercises, etc. The United Nations Educational Scientific and Cultural Organization UNESCO 2012a).

As for the Asia region which is one of the largest and most diverse region of the globe, ranging from countries as large as China and India, already the two largest mobile markets in the world, to a number of small islands and territories (GSMA Intelligence 2015). In this region, majority of people own and use mobile phones and devices. In 2010, according to ITU stats, there were nearly 70 mobile phone subscriptions per 100 inhabitants in this region (The United Nations Educational Scientific and Cultural Organization UNESCO 2012b). However, it has been shown in a study conducted by UNESCO in 08 Asian countries (China, Indonesia, Japan, Malaysia, South Korea, Singapore, Taiwan and Thailand) that few projects and policies tied specifically to the use of mobile devices, particularly for education, exist (The United Nations Educational Scientific and Cultural Organization UNESCO 2012b), covering different countries and territories with a broad range of languages and cultures. Knowing that there is a tremendous diversity of the Asian countries, the UNESCO review reveals that these countries tend generally to fall into one of the following three main categories in terms of their engagement with mobile learning: "(1) countries with a mature mobile market, high penetration of mobile phones and strong ICT infrastructure: Mobile learning is included under the broad context of national-level ICT policies. (2) Countries with a growing mobile market, medium to high penetration of mobile phones and basic ICT infrastructure: Mobile phones are used for distance learning and in informal learning contexts. (3) Countries with an emerging mobile market, low to medium penetration of mobile phones and weak or basic ICT infrastructure : Mobile learning activities are scarce" (The United Nations Educational Scientific and Cultural Organization UNESCO 2012b).

Regarding the North America region, many educational institutions provide mobile devices for all students, to ensure equity and consistent access to mobile opportunities. In the United States, two main initiatives deserve to be quoted: the first, Qualcomm's Wireless Reach initiative, created in 2006, and the second is the FCC's Learning On-the-Go pilot programme, launched in 2010 (The United Nations Educational Scientific and Cultural Organization UNESCO 2012c). Both programmes are BYOT (Bring Your Own Technology) based initiatives aiming to support and encourage students to experience new modes of active learning by bringing and using their mobile devices at school and outside (Quillen 2011).

Many other broader mobile initiatives also exist with the aim to promote mobile applications and content creation via training, education, and competitions. As examples, we can cite the Youth Mobile ${ }^{3}$ project, one of the UNESCO's projects for sustainable development via education and technologies. With such initiative, UNESCO and partners strive to provide young people with the high-level skills and confidence to develop, promote, and sell locally relevant mobile apps that solve local issues of sustainable development and provide employment.

Imagine $\mathrm{Cup}^{4}$ is another project led by Microsoft Company. It is an international student technology competition offering to students all over the world the chance to get involved in mobile technology field and to win awards. Many Arab students are participating regularly in this competition and won several times many prices. Unfortunately, there is no similar Pan-Arab initiative to promote mobile apps and content targeting Arab people, except some local projects that did not yet gain a Pan-Arab dimension and mostly are not comprehensive. For example, we can cite the Arab Mobile Application Challenge (AMAC) ${ }^{5}$ initiative, which aims to promote mobile application development in Arab countries, but did not yet cover capacity building activities and technical issues.

As can be seen, the mobile technology realm has witnessed amazing advancements and synergistic opportunities with several priority sectors such as education and social and economic development. In light of drivers and benefits of mobile technologies, there is a real need to create initiatives and policies in order to highlight and benefit from the multiple advantages of this technology and its positive impact on education and employability and other sectors. Nevertheless, in the Arab region, there is a luck of such regional and common Pan Arab initiatives and/or policies to promote mobile technology harvesting its potential for education and employability and other priority sectors, and to overcome barriers 
(mostly related to global markets, Arabic Language, technical infrastructure, skills, regulation, etc.). Therefore, ALECSO has launched a regional comprehensive initiative in order to go a step further in the massive use and production of Arab mobile apps and content in education, culture, science and serious games. This initiative spans some required aspects related to the Mobile ecosystem namely the technical infrastructure, soft and tools, markets, trainings, incentives, etc.

\section{ALECSO mobile apps initiative}

As shown previously, in spite of an increasing techie Arab young population, highly interested and even addicted to mobile devices and smartphones, the number of Arabic language based mobile applications developed so far is still few. Endeavoring to address this issue, ALECSO organization has proposed a comprehensive and strategic initiative ${ }^{6}$ (ALECSOApps) aiming to provide the necessary technological and institutional environment for the promotion of an emerging digital creative Arab Mobile industry, related to the fields of education, culture, science and serious games (Jemni and Khribi 2016a). The ALECSO mobile apps initiative has started in 2015 and it is still running. This initiative involves 22 Arab countries members in the Arab league. Some partners including ministries of education and ICT, universities, and mobile phone and telecommunication companies in the Arab region are involved likewise. In general, the key objectives of that initiative, ranging from the raising awareness and capacity building features to the implementation of appropriate tools and technical environment and financial boost, cover the following:

- Taking advantage from mobile technologies since it can deliver many important social and economic benefits in the region, especially by improving access to education, and culture and financial services and healthcare and other priority sectors;

- Supporting the mobile industry in the Arab region, which will yield economic growth and entrepreneurship, and major improvements in education, financial, healthcare, social, etc. services;

- Preparing the digital ecosystem for the new mobile opportunities, particularly in the areas of job creation, and business prospects, and economic growth.

- Offering to the 400 million Arab population a Pan-Arab wide market with advanced technical infrastructure for the hosting and exchanging of a selection of Arab mobile Apps, with new business models and opportunities.
- Introducing mobile technologies to all in the Arab region through a specific training program and material available in several learning modes (face to face, online, blended, etc.) for a large spectrum of learners (from newbies to experts);

- Encouraging the Arab youth to be more competitive and innovative by offering possibilities to reach specific mobile learning programs providing required knowledge and skills;

- Providing a suitable technical environment for developing and testing and hosting mobile applications;

- Promoting Mobile Learning in the Arab region, especially by empowering mobile learning opportunities by proposing low-cost and easy-toimplement solutions to make education more accessible to all students;

- Motivating the Arab mobile developers and give them the opportunity to create and innovate by organizing a Pan Arab wide mobile competition to generate more profound mobile innovations and select annually the best apps in the fields of education, culture, science and serious games.

Given these points, many projects and activities have been proposed to let the initiative come to fruition quickly and efficiently. Indeed, the following flagship projects ${ }^{7}$ are delivered (Jemni et al. 2015):

- ALECSO Apps Store;

- ALECSO Apps Editor;

- ALECSO Apps Award,

- ALECSO Apps training programs.

\section{ALECSO apps store}

The ALECSO Apps Store is a web-based application intended for hosting and gathering Arab Mobile Applications (Fig. 1). This platform is also installable on supported mobile devices that run Android OS. ${ }^{8}$ This Arab marketplace provides the opportunity to Arab developers involved in Mobile Technology to innovate and to share their creations without any fees, restrictions or barriers. To ensure a good quality of applications on the store, a strict publishing process is adopted. Indeed, before publishing a submitted application, a team of experts must assess it and ensure the checkup phase. The architecture of the ALECSO Apps store platform is designed to ensure a high quality of services. It is composed of two main modules hosted on two different servers. The first module is web based and it ensures the management of the web interface of the store. The second module is Cloud based. It ensures all features that need processing the binary files such as metadata, size, signature, etc. This architecture is intended to be gradually entirely cloud based. 

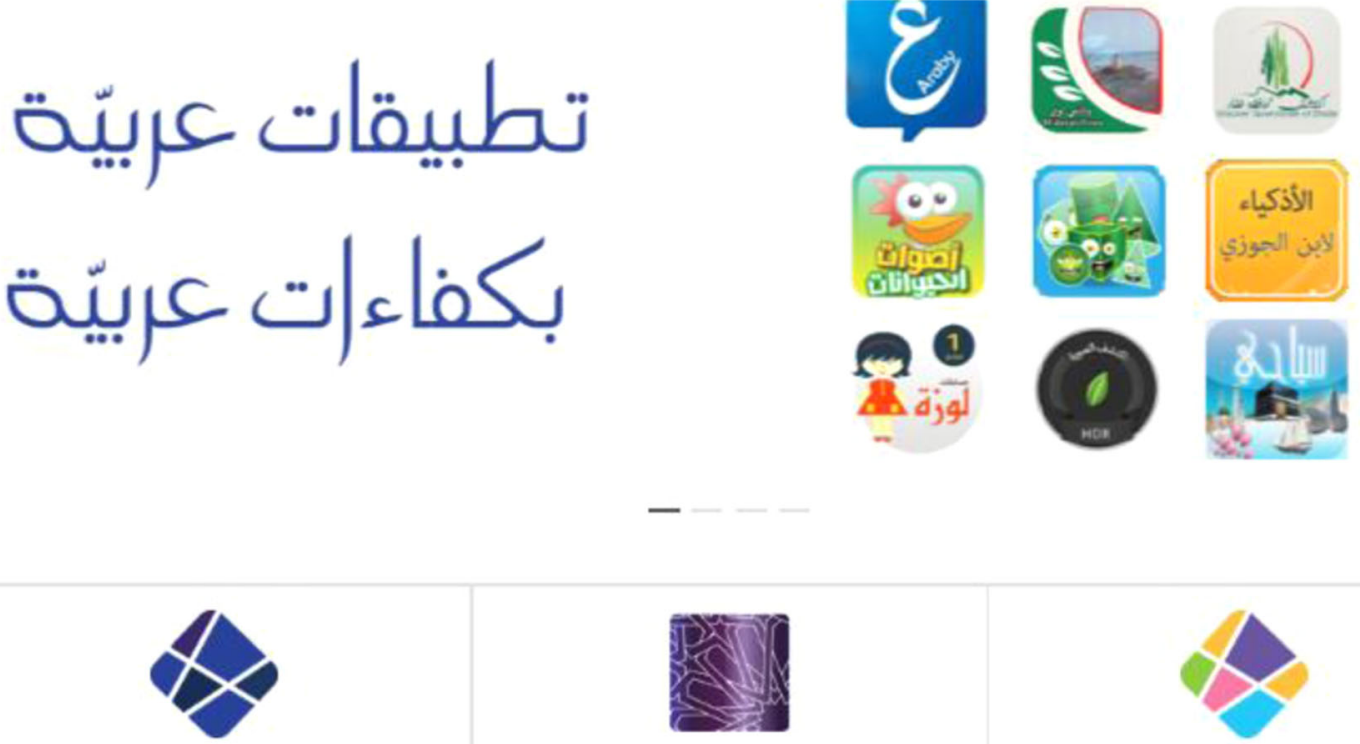

AlecsoApps Store

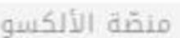

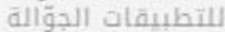
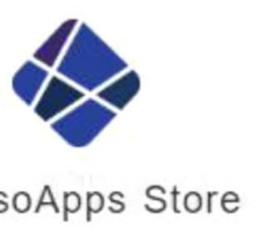

AlecsoApps Awards

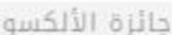

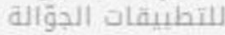
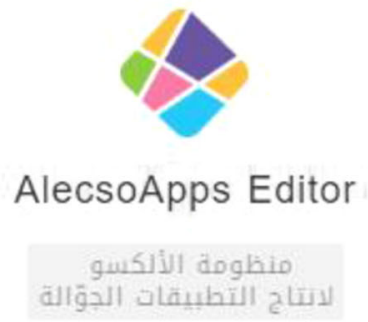

آخر التطبيقات المضافة


Fig. 1 The ALECSO Apps Store

\section{ALECSO apps editor}

Recently, many developers are relying more and more on hybrid techniques for the development of mobile applications. In fact, they are using generally combination of web languages (e.g. HTML5, JavaScript and CSS3). To do so, developers tend to use hybrid development tools. Phonegap ${ }^{9}$ is one of the most well-known tool which allows the compilation of web source code embedded in mobile apps, using the potential of mobile devices and their features, and can be run for Android or iOS or Windows Phone operating system (Shotts 2013).

\section{Review of cross-platform development}

Currently, with over than four millions of existing mobile applications in leading app stores, developers are facing new daily challenges to innovate, create, develop, deploy, manage, publish, and disseminate mobile applications. The complexity of such situation makes it difficult to estimate the development's cost and time of mobile applications suitable at least to the most used and leading app stores and related platforms. The Cross Platform Mobile App Development's concept has tremendously simplified this process since developers can 
run seamlessly their apps across multiple mobile platforms. So it looks like "one code fits all" since it is possible to code an application that can be run on multiple platforms without tweaking the code belonging to each obtained native version.

Many tools are available to develop flexible mobile applications with seamless compatibility, like: PhoneGap, Appcelerator, Sencha Touch, MonoSync, Corona, Adobe Air, Xamarin, RhoMobile, etc.

- PhoneGap (Apache Cordova): an open source tool that enables developers to create mobile applications compatible with Android, iPhone, iPad, Palm, BlackBerry, and Symbian.

- Sencha Touch: HTML5 based cross platform framework that provides fully functional APIs and offers a component based approach for building mobile applications.

- MonoSync: This framework supports $\mathrm{C}$ and $\mathrm{C}++$, PHP, Java, JavaScript, Python, and Ruby on Rails for mobile development apps.

To be able to choose the most suitable tool a developer can lean on, besides local needs and requirements, a well-defined list of criteria should be taken into consideration. Those criteria cover, among others, what follows: cost, platform support, access to hardware and device API's, performance, architecture, technical aspects, leaving curve, productivity, user interface, usability, efficiency, supported languages, testing tools and debugging, etc (Holzer and Ondrus 2011).

\section{Discussion}

The cross platform development technological ground is constantly shifting with each tool introducing new functionalities and facilities to the communities of developers and users. In the ALECSO Apps project, one of the major criteria by which we are concerned is related to the no support of RTL (Right to Left) languages, and especially Arab language, in addition to other technical matters related mainly to the multi-users, multi-tasks and compilation issues. There is no doubt that all the aforementioned existing cross-platform mobile development tools are actually covering most of the required components to create apps (such as pages creation, media integration, rich text editors, app personalization, etc.), but they don't support Arab language neither in the interface nor in the content of applications. Whereas our main need, in the ALECSO Apps project, is to provide non-expert users with a comprehensive framework enabling them to create and build intuitively Arabic language based mobile apps.

\section{ALECSO apps editor: arab-based cross-platform apps devel- opment studio}

The ALECSO Apps Editor (Fig. 2) is a web based platform that provides an environment of Rapid Application Development (RAD) (James 1991). It represents a comprehensive mobile applications development Studio. It enables users to create their applications in few intuitive steps. The Editor is mainly proposed for people whom do not master programming languages such as JAVA or objective $C$ and includes a real time previewer based on WYSIWYG. The main specificity of ALECSO Apps editor is its ability to build Arabic mobile apps based on a set of embedded easy-to-use tools. Those tools cover actually the most requested components by the Arabic community and among them, we can mention the pages creation tool, the rich text editor, the video gallery tool and photo album tool (Fig. 3). Since this Editor is based on web technologies like HTML5, CSS3 and JavaScript, it becomes easy for users to integrate new plug-ins in

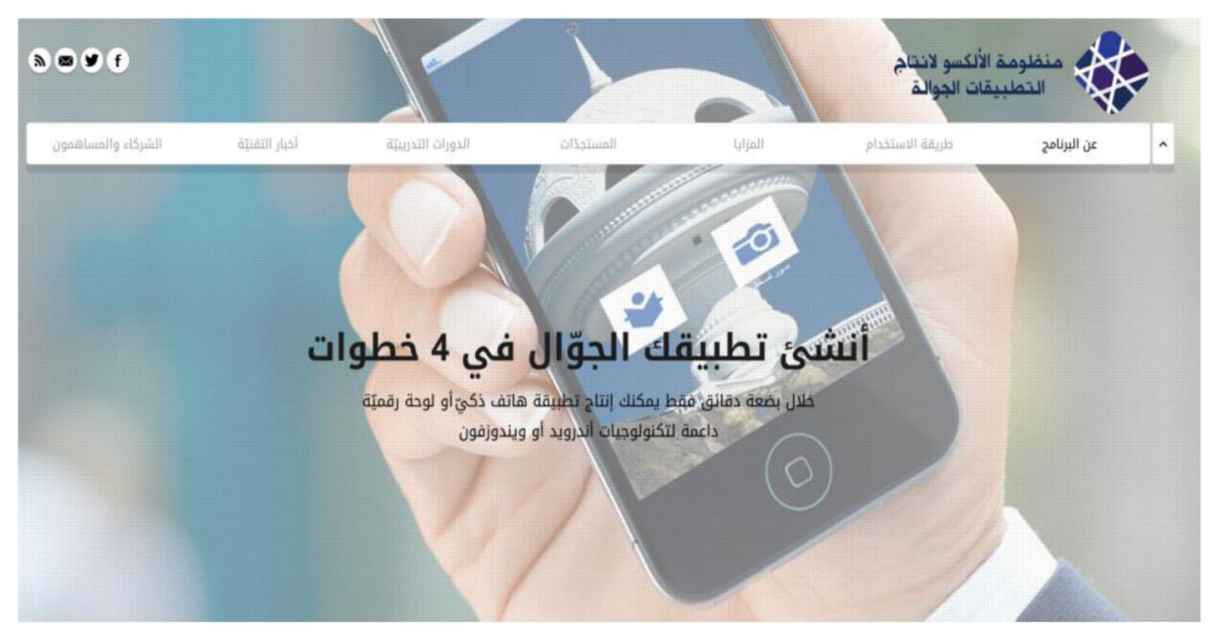

Fig. 2 The ALECSO Apps Editor 


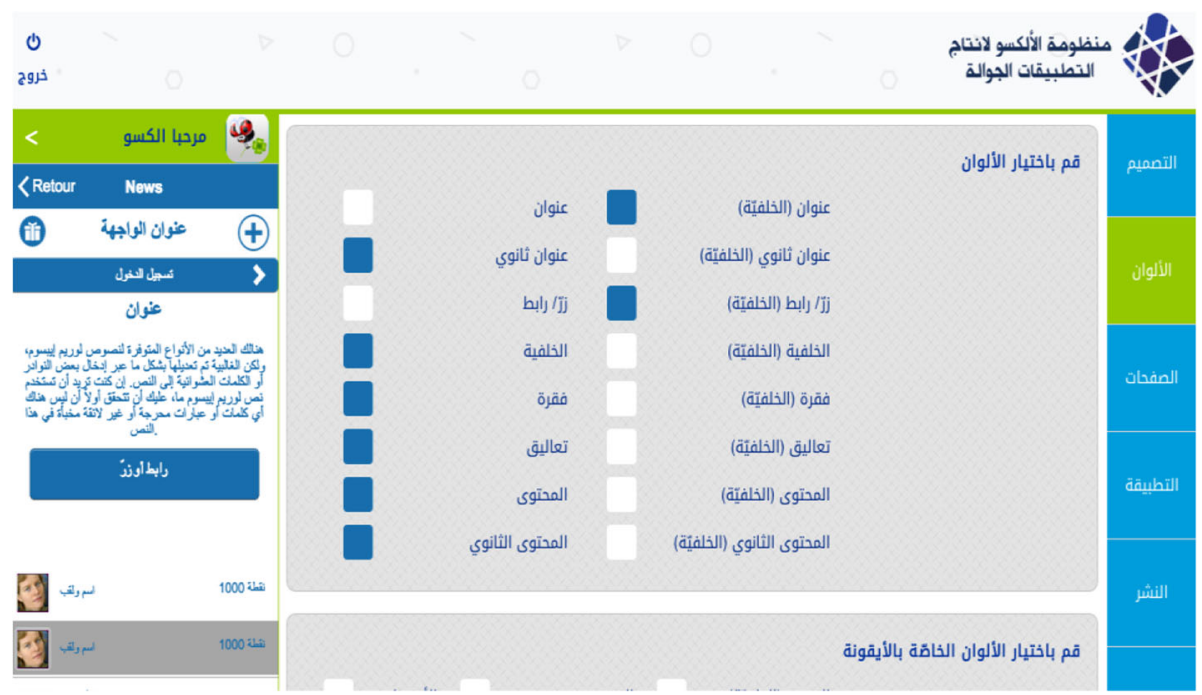

Fig. 3 Creation of mobile applications intuitively in few steps

addition to the pre-built plugins provided by default. The process of generating the final mobile app is done in a transparent way where the user receives the compiled app according to the target platform that she/he specifies. Therefore, the generated app is a hybrid mobile app that takes the advantages at a time of web and native applications. The application is subsequently able to access to sensors and other hardware features available on mobile devices such as the rear and front cameras, the GPS and the gyroscope, etc. The technical details about the Editor's software architecture will be presented in the next section.
As explained above, applications generated by the ALECSO Apps Editor are able to run on any device since they are built using web technologies and wrapped and compiled with Apache Cordova. Thus, these applications can access to all native device capabilities and features. Thereby, advanced users can then use the source code of the app and add more plugins in order to enrich the application with native functionalities.

Architecture overview The Editor connects the user interface with the back-end system. This system adopts a very flexible visual approach to provide

\begin{tabular}{|c|c|c|}
\hline \multicolumn{3}{|c|}{ EDITOR'S FRONT } \\
\hline \multirow{3}{*}{\multicolumn{2}{|c|}{$\begin{array}{c}\text { User Interface } \\
\text { Pictures Processing Tool } \\
\text { Source Code Console }\end{array}$}} & Components \\
\hline & & Templates \\
\hline & & Plugins \\
\hline \multicolumn{3}{|c|}{ LIBRARIES } \\
\hline \multirow{2}{*}{$\begin{array}{l}\text { HTML, CSS, } \\
\text { JavaScript }\end{array}$} & \multirow{2}{*}{$\begin{array}{c}\text { Jquery Mobile, } \\
\text { AngularJS, Bootstrap }\end{array}$} & Apache Cordova \\
\hline & & Native Plugins \\
\hline \multicolumn{3}{|c|}{ BACK-END SYSTEM } \\
\hline Hosting & Database & Compiler \\
\hline \multicolumn{3}{|c|}{ INTEGRATION FRAMEWORK } \\
\hline \multicolumn{2}{|c|}{ Feeds } & Integration \\
\hline
\end{tabular}

Fig. 4 ALECSO Apps Editor Architecture 
final users with high level of customization. Indeed, users can benefit from the web-based visual platform to collaborate and share the application view in order to tune the application simultaneously. As illustrated in Fig. 4, the platform of the editor is based on four layers: Editor Front, Libraries, Backend system and the integration framework. The Front is the user interface service which allows developers to select the provided components and build the application's pages. We should notice that other third-party tools like online picture processing are provided as well. Moreover, a set of predefined templates and plugins are provided, otherwise the developer can customize and/or add new developed templates.

In the next layer, we find libraries that represent mainly the basic web technologies that will be used to render the web version of the mobile app. The apache Cordova, which is a Javascript library, allows the creation of plugins ensuring access to native features like storage, network, camera, etc. The following layer is the Back-end system, which is the engine of the Editor. In Fact, this part of the platform represents the components used to manage users' accounts, authentication as well as apps compiling. As for the last layer, it represents the integration functionalities gathering the most known APIs which allow developers to embed external data resources natively. Indeed adding a map from google, a video from YouTube or a feed from any website is possible thanks to the integration framework.

Infrastructure Actually, the Editor is deployed on a cloud based-infrastructure. Two main instances are dedicated to both the Editor's Front and the Editor's compiler. To guarantee a high level of availability the editor's platform is built behind a very scalable Cloud DNS lookup service. This service, called "Cloud Front" automatically roots the traffic to zone2 (Ireland) where the whole platform is cloned. The compiler is an intensive service in term of computation requirements. It allows the editor's Front to convert the generated apps form HTML5 into WP8 and Android binaries, which are composed, of native containers that embed Web views. The average time necessary to compile an application is around $45 \mathrm{~s}$ on a single instance. The compilation average time can cause bottlenecks especially during rush hours and simultaneous access (e.g. during training sessions). Subsequently, a mechanism of auto scaling and load balancing are necessary in order to handle excessive simultaneous calls to the compilation process. The detailed infrastructure is shown in the following figure (Fig. 5).

\section{ALECSO apps award}

The ALECSO Apps Award is an annual Pan-Arab competition aiming to motivate and encourage Arab developers to meet high-level standards in term of Mobile Applications quality, innovation and Entrepreneurship. The competition is set over two stages: The first stage is national, lead and held locally in each Arab countries to select the best national Mobile Applications, in the areas of Education, Culture, Science and serious games. The second stage is rather at a Pan-Arabic level: wining applications are selected among those getting successfully the first round. The amount of the ALECSO Apps

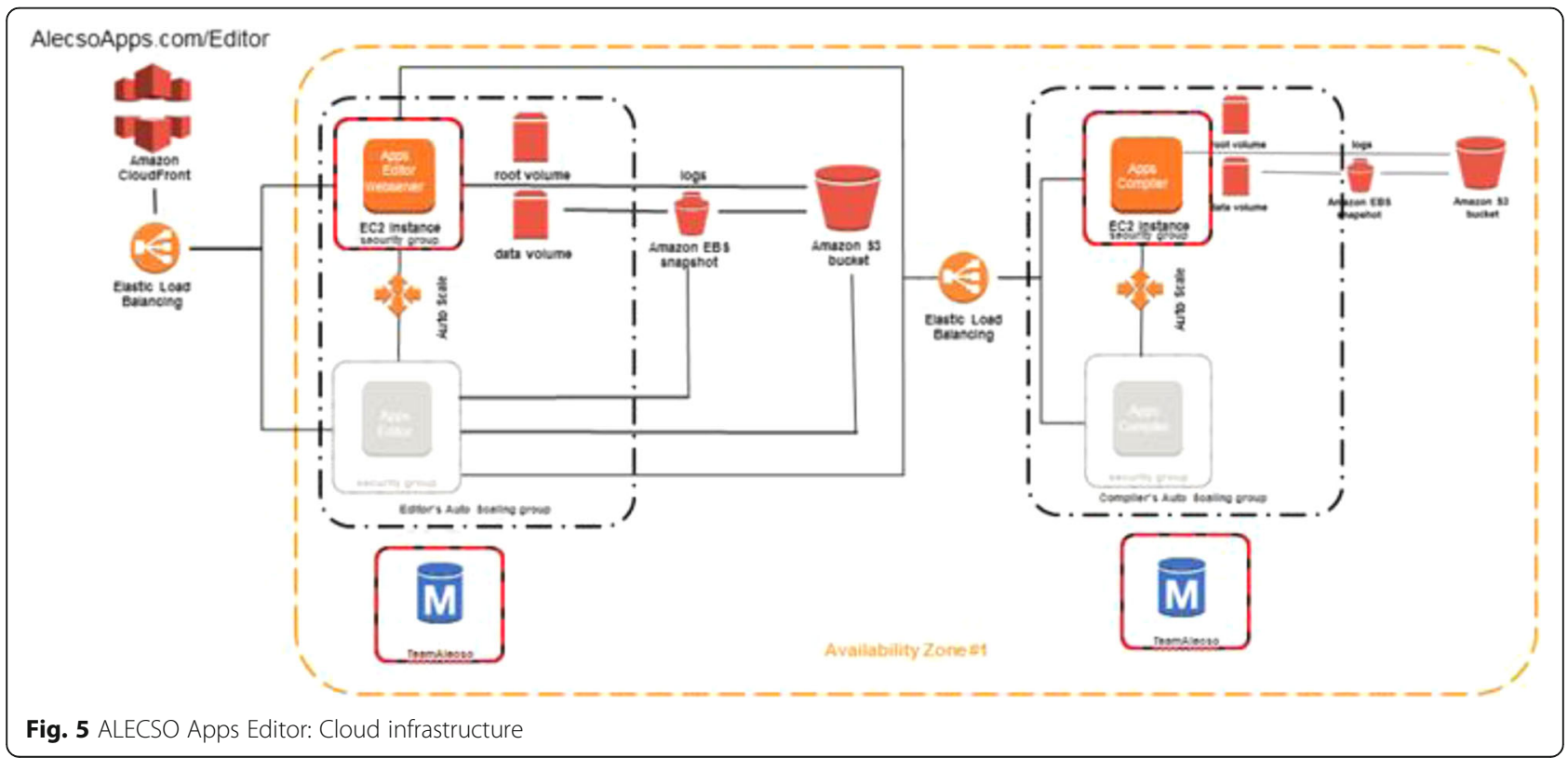


Awards is about 50000 \$US. The submission and the evaluation of applications are done online (Fig. 6).

Many reviewers from different Arab countries participated in the evaluation of each application. The evaluation is based on many criteria such as innovation, creativity, features, content, user interfaces, technology and even application packaging. During this phase, Internet users can choose and so nominate the winner of the audience award applied through the voting system. The first edition of the ALECSO APPS Award was held in Qatar (Doha 2015), the second edition was held in UAE (Dubai 2016), and the third edition is going to be held in Tunisia (Tunis 2017).

\section{ALECSO apps training programs}

In order to introduce adults and youth people in the Arab region to the mobile technologies realm, and build, and reinforce capacities in Mobile Apps development, ALECSO offers varied training programs ranging from short workshops, to specific and advanced mobile development programmes for a wide spectrum of trainees from all the Arab countries. ALECSO Apps training programs provide trainees with continuous and consistent access to training, technology, and networks. The educational resources, material and activities used during training workshops, and covering the required skills for the development of Mobile Apps, are available online via the ALECSO online training platform. ${ }^{10}$ So that discussions and interactions via the platform can continue after the official duration of workshops. Trainees are mainly teachers, students and developers from Arab countries. The ALECSO Apps training Programs are ensured basically via three modes:

- Face to face training workshops using e-learning facilities to enhance the quality of training;

- Online learning, in the form of Small Private Online Courses (SPOCs) and Massive Open Online Courses (MOOCs);

- Blended learning, in partnership with Academic and industrial partners in the region.

A set of learning and training online environments with necessary technical cloud computing based infrastructure are used to ensure these training programs, including e-learning platforms, SPOCs and MOOCs platforms (Jemni and Khribi 2016), and a compiler which is a specific tool allowing the conversion of generated HTML5 apps to specific mobile OS format binaries (Android, Window Phone, etc.).

As for training material, and as we opt for a mobile cross-platform development, the material focuses mainly on the creation of cross platform hybrid mobile apps with native look using known technologies i.e. HTM5, CSS3 and JavaScript. So that all the trainees whether they are web developers or beginner in web development, can easily pitch into mobile apps development. Moreover, with hybrid apps development, developers can write the hybrid mobile app once and run it on every platform without any extra efforts. The HTML5 UI frameworks provide many components that help a lot in the design of responsive web app that fits all

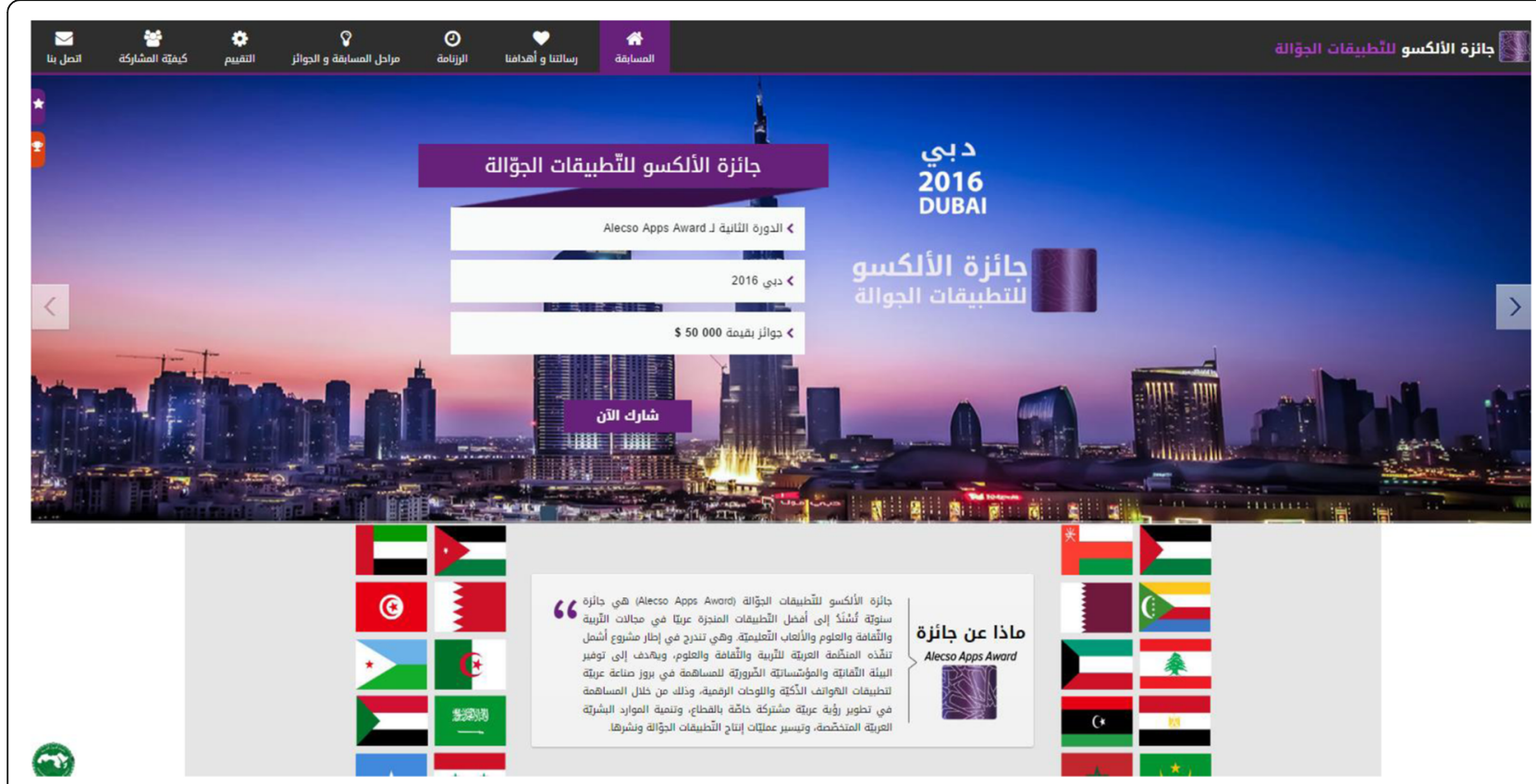

Fig. 6 The ALECSO Apps Award web site 
devices' screens, and it is also possible to access all native hardware components such as Camera or GPS thanks to JavaScript APIs, whether already existing like those offered by Cordova (PhoneGap) or newly written and compiled. All the educational resources and environments used in this project are under open license.

Furthermore, a specific training programme (called ALECSO M-Developer) based on blended learning was launched earlier in 2015. The pilot experience of this programme was held in Tunisia with the partnership of the Tunisian Ministry of communication technologies and digital economy, and Tunisian universities, aiming to train 500 students enrolled in graduating class or newly graduated, on mobile apps development. The programme includes a training of Trainers workshop, a face-to-face training phase, an online learning phase via a dedicated SPOC and a coaching phase to define the Apps' ToRs and to develop and finally deliver and publish the Apps. As a second stage, ALECSO organization intend to duplicate this pilot project for Arab countries expressing an interest in disseminating and localizing mobile technologies.

\section{M-Developer programme}

The M-Developer Program is a specific hybrid training program whose first edition has just been finished (December 2016). The pilot experience of this programme initiated by ALECSO, is the result of a fruitful and successful collaboration between the organization and the Tunisian Ministry of Technologies of Communication and Digital Economy. Co-financed and monitored by both entities, the project aims to build a capacity in the realm of Mobile technology for a young audience of regular students enrolled in Tunisian universities. ALECSO is in charge of designing and implementing the program. For this purpose, a specific hybrid model of training is setup taking into consideration a number of constraints related mainly to students' attendance, technical aspect of the course, etc., and taking full advantages of ICT use in education. In fact, 500 Tunisian students among those enrolled in graduating class or newly graduated, belonging to the ICT major, are selected to attend such a specific training on mobile apps development (it is worth noting this number was raised later to 569 enrolled students). These students are, then, grouped into 20 cohorts (Fig. 7), each of which is taken up by an instructor who is in charge of all the training phases, ranging from face to face workshops, to online training and coaching sessions. For this purpose, a set of platforms and tools are used in order to offer appropriate learning experience to students.

As a second stage, ALECSO organization intends to duplicate this pilot project for other Arab countries expressing an interest in disseminating and localizing mobile technologies, and especially, preparing new generations of skilled and competitive mobile developers in the Arab region.

\section{M-Developer core activities}

The program includes primarily five milestones in terms of training:

- Training of trainers' workshop, with the aim of providing to trainers a profound description of the program and its specificities, and to explore deeply the training material (Fig. 8). Twenty instructors among interested teachers in Tunisian universities, experts in the field of ICT and mobile development, are hired as contractual trainers under $\mathrm{M}$-Developer program to ensure all the training phases for a duration of six months.

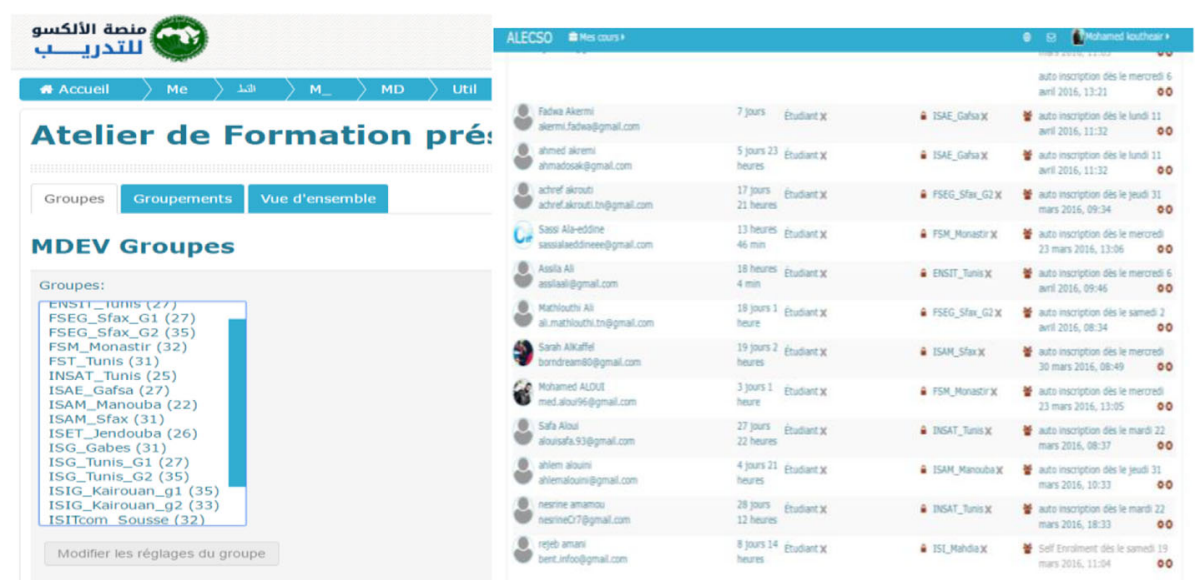

Fig. 7 Registered students and cohorts 


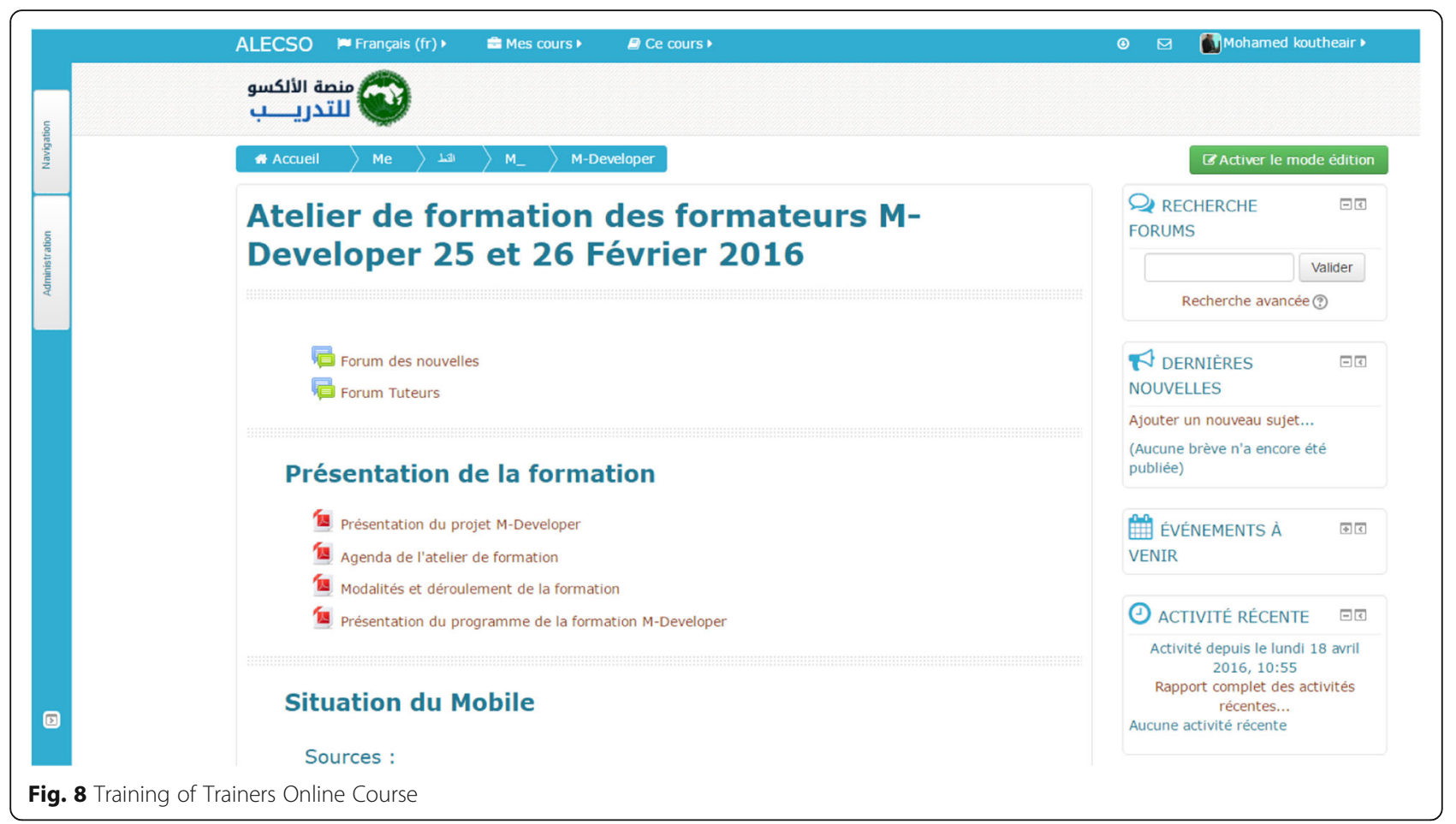

- Face-to-face phase training, each trainer is asked to organize a five-days face-to-face training workshop for his/her group of students. Even in these face-to-face workshops, an online learning platform is used in order to host the training material designed and developed by ALECSO to conduct in total twenty five-days face-to-face workshops throughout Tunisian universities and Tunisian regions, with several possibilities to use communication and collaborative tools whether between students themselves and/or with instructors (Fig. 9). It is to be noted that first face-to-face workshops are three-days based. Then, there oneday workshop held in the mid of the online phase and finally, last one-day workshops at the end of the online phase.

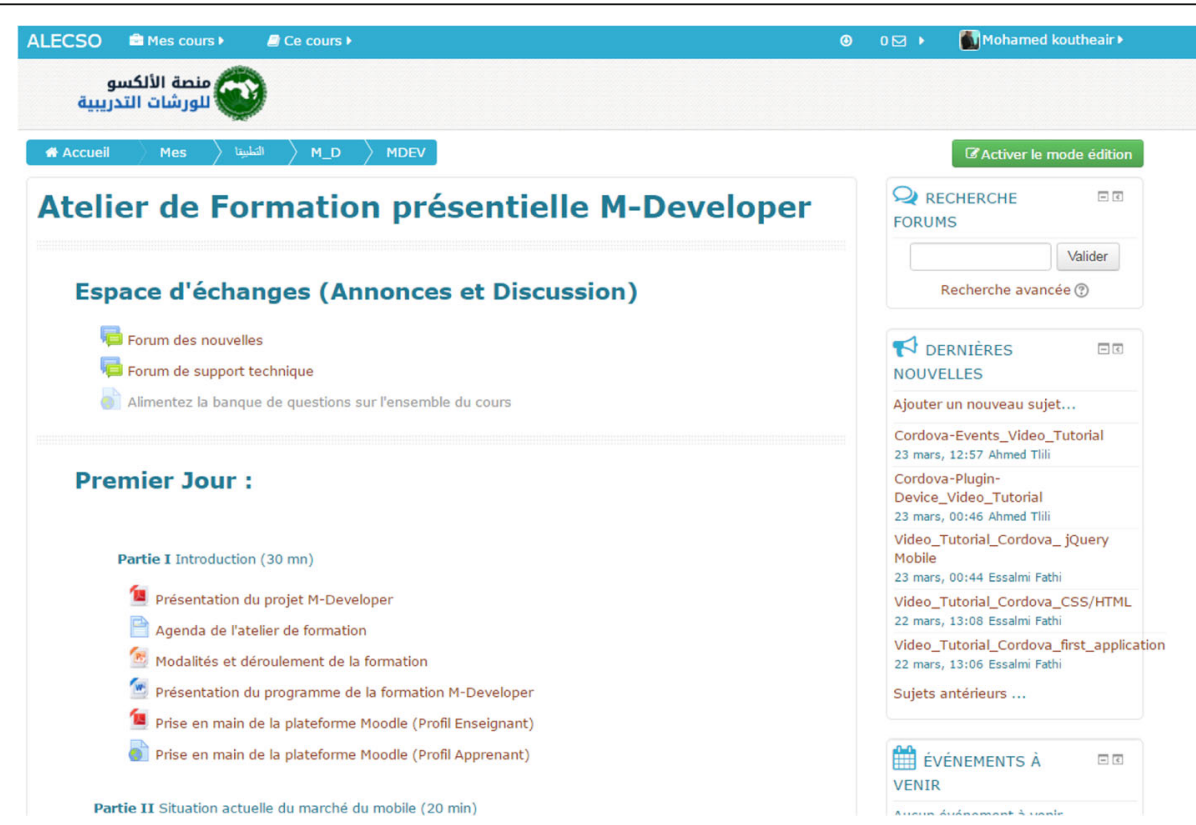

Fig. 9 ALECSO Online Training Platform 
- Online phase via a dedicated Small Private Online Course SPOC, this phase lasts six weeks during which students access to several videos and different learning resources. They are also asked to make Quizzes and assignments. During this phase, students are supervised and guided by their instructors (tutors).

- First coaching phase lasting one month with the aim to help students finding appropriate project's ideas and defining properly their mobile apps' ToRs;

- Second coaching phase lasting one month aiming to develop and finally deliver and publish the required mobile apps.

\section{M-Developer SPOC}

It is noteworthy that M-Developer project represents actually a kind of results-based project, which means that at the end of the program, we should get as concrete outcomes, besides the students' completion of all training phases, dozens of decent mobile apps developed and published. Moreover, given that the program was initially designed for a relatively small number of regular students throughout Tunisian universities, gathered in several groups, with focus on the instructor role during the training phases, This would avoid somehow students' potential dropouts or disinterest. It was therefore agreed that the online phase would be ensured using a SPOC course type instead of MOOC, even though in terms of content, there are almost no differences between both types, except the "massiveness" and "openness" dimensions. Indeed, the M-Developer SPOC is only accessible for those who are registered via the M-Developer program, and thus not open for all, and the number of registered students is relatively small, added to that, gathered in tiny groups, with an instructor for each.

\section{M-Developer syllabus}

The SPOC lasts six weeks, it is a 42 h equivalent semester module in university. Each week includes specific tasks to ensure progressively week by week the completion of the entire training phase and then be able to step ahead to the next stage in the Program. During each week of the SPOC, students are asked to get through the following (Fig. 10):

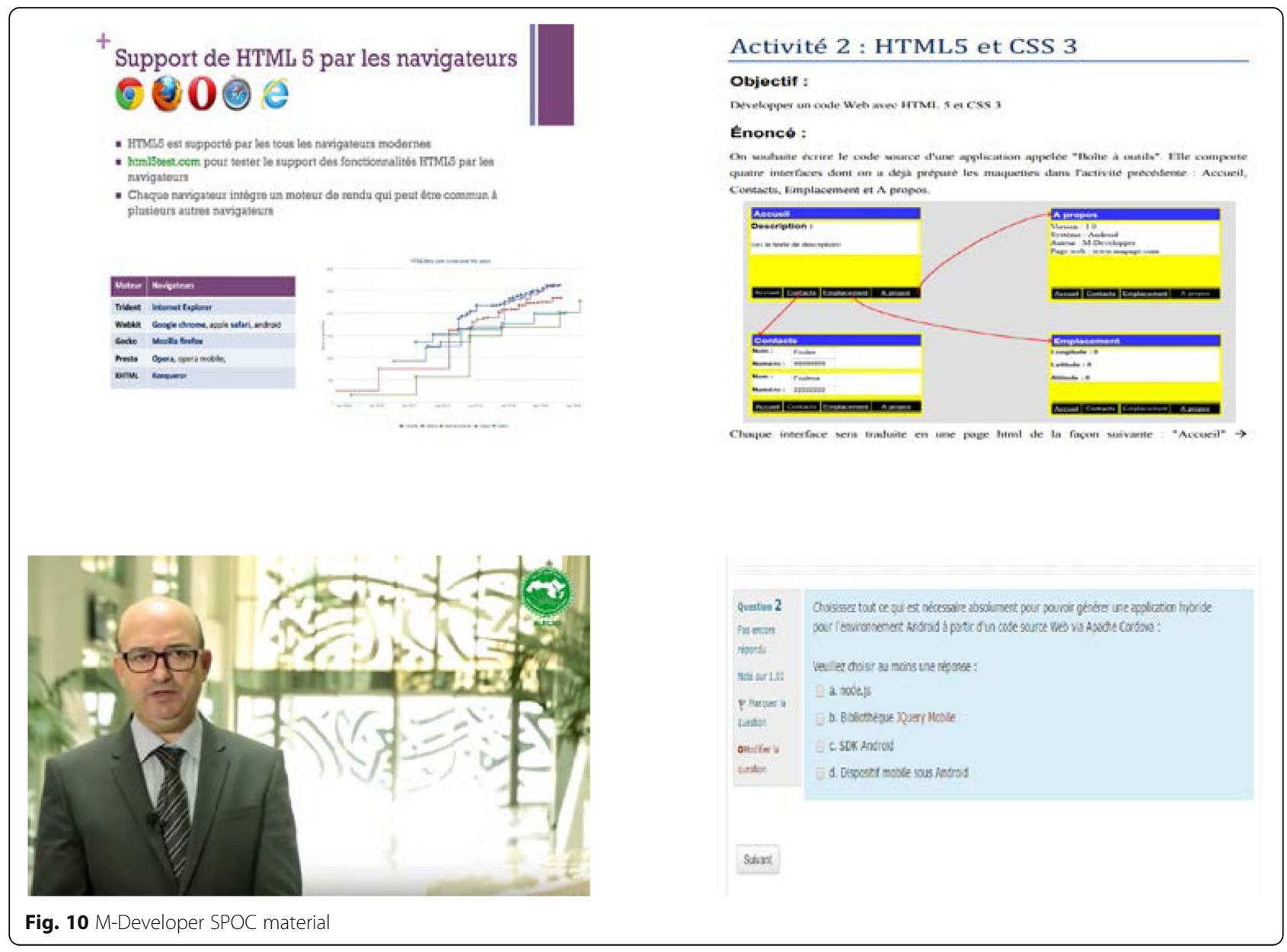


- Access available short video lectures,

- Explore available presentations, documents, tutorials, and extra learning resources if any,

- Answer to Quizzes (involving corrections to better understand learning material),

- Make assignments, problem sets, and submission in due date,

- Pass a final Quiz test (weekly and at the end of the SPOC).

Obviously, students can interact with each other, ask for clarifications, launch discussions, and put questions using available Forums within the course. Basically, lecture topics are organized among the six-weeks SPOC as follows:

- Week 1 : o Welcome address and Introductory remarks o M-Developer Syllabus o Pitching Project Ideas o Mobile Apps Opportunities o Status of Mobile technology o Mobile Operating Systems

- Week 2:

o Mobile Development

o Native and Hybrid Programming

o Design and Interfaces

- Week 3:

o Web Programming Languages HTML5, CSS3, JavaScript, JQuery

- Mobile Apps Ads

- Week 4:

o Cordova Environment

- Week 5:

o Interactions with eternal services (Client-server Architecture, JSON, Examples, etc.)

- Week 6: o Cordova Plugins

o Mobile Apps Sample Project

o Mobile Apps publishing

o Business Models

\section{Grading policy}

In this SPOC course, as shown above, there are varied types of assignments. The obtained final grade is weighted average of the following:

- Problem sets : 40\%

- Weekly Quiz : 30\%

- Final Quiz: 30\%

In order to earn a certificate at the end of the SPOC, students must pass the course with a grade of $\mathrm{C}$ or better. The following grading breakdown will apply:
$->=80 \%: \mathrm{A}$

$->=70 \%: \mathrm{B}$

$->=60 \%: \mathrm{C}$

In order to earn a certificate at the end of the Whole M-Developer Programme, students must:

- Attend all the training phases,

- Earn a SPOC certificate,

- Prepare and pitch a project idea,

- Prepare and submit the project's ToRs,

- Develop and publish the developed mobile Application.

It is noteworthy that, following the new ALECSO vision to open up digital content and training material, all developed learning resources are planned to be openly licensed using creative common licenses (Butcher 2011).

\section{Use and impact of the ALECSO APPs initiative}

Despite being launched in 2015, the ALECSO mobile apps initiative is still alive and well progressing, and all relevant stakeholders from many Arab countries have adhered to its values ever since. Moreover, many contacts have been initiated with international organizations, associations and companies working in the area of mobile technologies and telecommunication with the aim to create partnerships and to collaborate in order to enhance the quality of projects and to enlarge the scope of the initiative. In this respect, we have networked with GSMA aspiring to involve ALECSO APPs Awards within the Global Mobile Awards and to extend the competition policy accordingly. Furthermore, discussions with a couple of known companies working in the field of mobile technologies are ongoing, these companies are interested in the installation and use of the ALECSO APPs store initially on their smartphones, which will offer fruitful commercial opportunities to improve their sales rate and thus penetration in the Arab market (more than 400 million users). In the same vein, ALECSO is participating in the UNESCO mobile learning week held in March of each year in the UNESCO headquarters in Paris. By attending such international events, ALECSO aims to promote its Mobile Initiative, and consequently to expand international understandings of how such initiative can help, especially in the developing world, to open and enrich opportunities for developers (especially youth, girls, and women) to create and innovate. Which has an important impact on the growth of economy and entrepreneurship, and improvements in education, financial, healthcare, social, etc. services. In this context, other countries and regions were interested in this valuable experience, and how would it be possible to replicate it successfully overseas. 
Many relevant indicators are used to help out in measuring the scope of the use and impact of the initiative in Arab countries so far (e.g. enrolment, number of mobile apps in the ALECSO Apps store, usage stats, sentiment analysis, interactions in terms of comments and feedback and various requests, number of mobile apps submitted in each session of the ALECSO Apps Award, number of mobile apps created with the ALECSO Apps editor, number of training sessions, number of trainees, etc.). To this end, some dashboard interfaces were developed in order to give the required assistance in analyzing the use and impact of the initiative and all its components.

Regarding the ALECSO APPs store, current key figures indicate the availability of 458 mobile applications so far, more than $200 \mathrm{~K}$ downloads, more than $440 \mathrm{~K}$ views, more than $21 \mathrm{~K}$ ratings of different Apps, 756 comments, and 1898 registrants, as shown in Fig. 11, which reflects the progress status of mobile apps in the ALECSO APPs store and its use and dissemination since its creation recently.

As for the ALECSO APPS Award, the first edition of this unprecedented competition in the region was held in Qatar, and the closing ceremony was organized in Doha in January $14^{\text {th }}$ 2016. This first edition witnessed the submission of more than 1164 applications from 19 Arab countries via the Award web site. The reviewing committee composed of 13 members, whom are experts in the field from Arab countries, ensured a preliminary selection of the best submitted apps at a national level in the fields of education, culture, science and serious games. The result of this process provided 244 selected applications from 16 Arab countries. Eventually, during the closing ceremony, reviewers selected the best applications at a Pan-Arabic level. There were 10 chosen awarded apps, some of which were equally ranked and thus equally awarded. Winners are from 09 Arab countries: Qatar, KSA, UAE, Kuwait, Sudan, Tunisia, Jordan, Algeria, and Morocco. The second edition of the ALECSO Apps Award 2016 was held in Dubai, with the support and collaboration of the Telecommunications Regulatory Authority (TRA) in UAE. The Closing ceremony was held in November 20th 2016 during the UAE innovation week. This second edition has already witnessed the submission of more than 1300 applications from 19 Arab countries via the Award web site (Fig. 12). A preliminary selection of the submitted applications based on technical and relevance issues to the competition has led to some 516 appropriate mobile apps ready to be reviewed by the international board of experts in order to select best mobile apps in the fields of education, cultural, science and educational games, first, at national levels and then, at a Pan Arabic level. At the national level, 56 mobile apps were selected as the best apps. And during the closing ceremony, reviewers selected the best applications at a Pan-Arabic level i.e. among these 56 final candidates. There were 12 chosen awarded apps, some of which were equally ranked and thus equally awarded. Winners are from 10 Arab countries: Qatar, KSA, UAE, Palestine, Tunisia, Syria, Jordan, Egypt, Algeria, and Morocco.

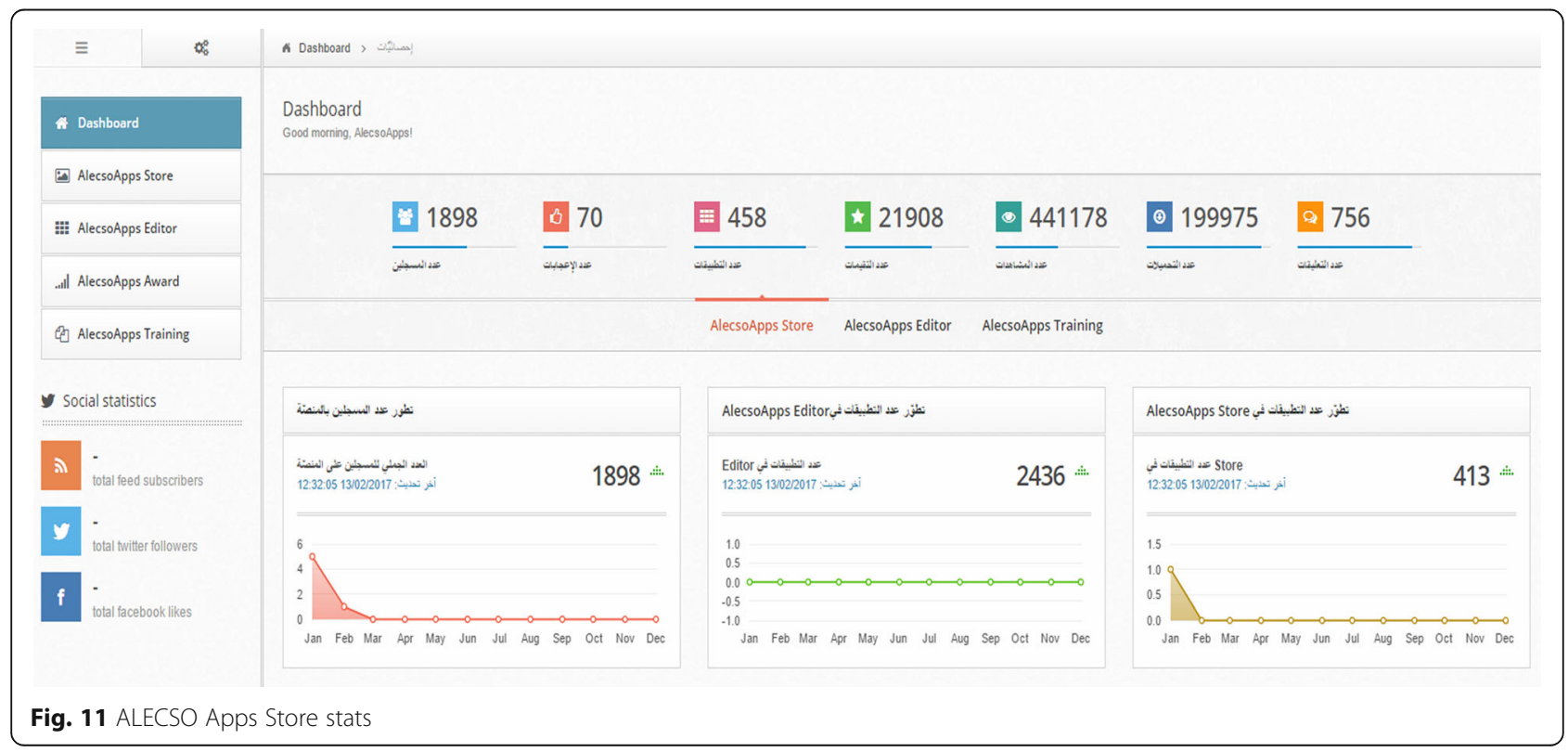




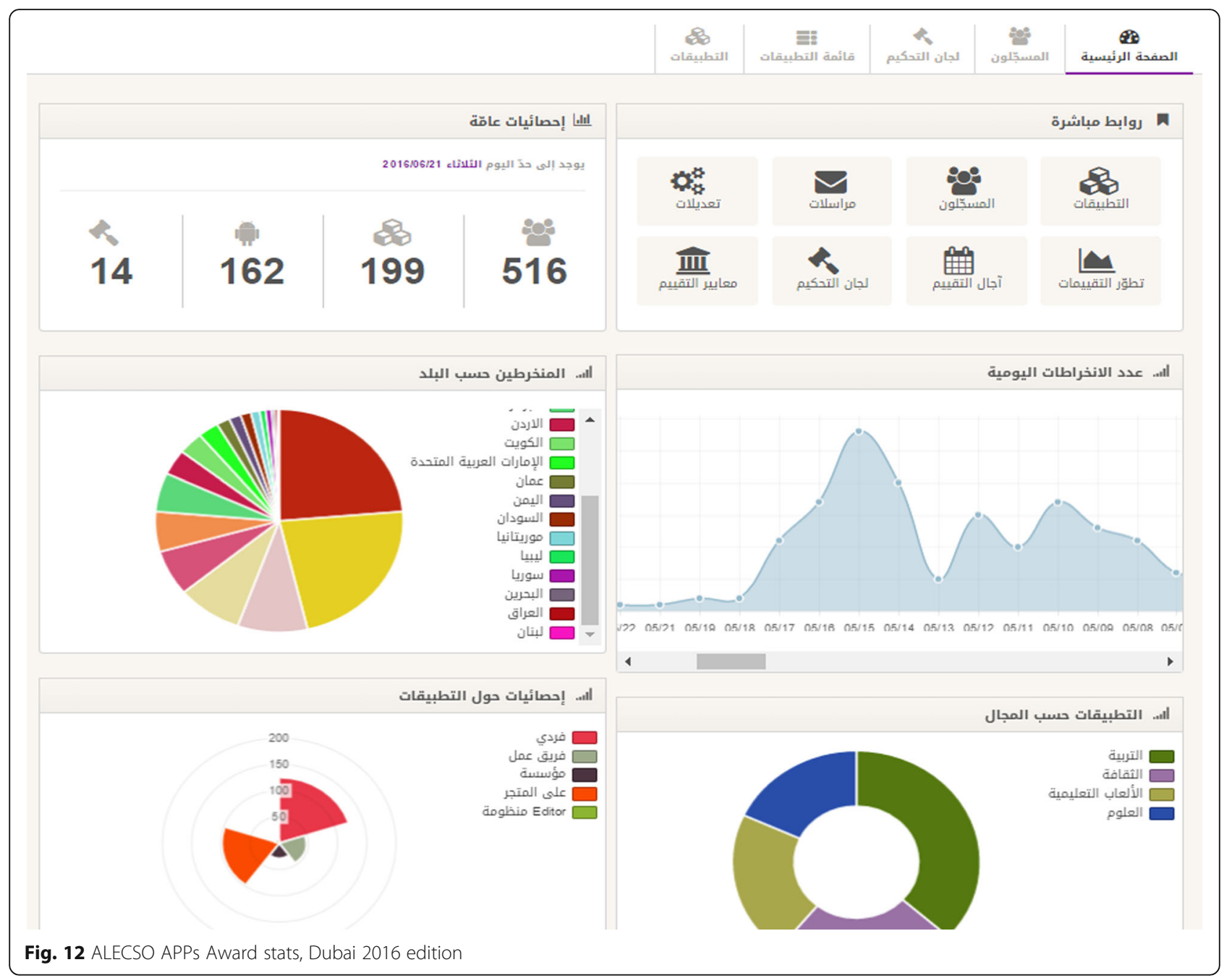

Moreover, a dedicated page to the ALECSO APPs Award is created on Facebook with more than 41000 subscribers so far (Fig. 13). Other social networks are used likewise in order to raise the spectrum of interested people and to promote more and more the initiative and all its components.

In regard to the ALECSO APPs Editor, which is used to build Arabic based mobile apps in few intuitive steps based on a set of embedded tools and environments, especially the compiler which allows the editor's Front to convert and generate apps form HTML5 into WP8 and Android binaries. The editor's front and back are mostly used in the context of training sessions. There are actually 1546 generated mobile apps, and some 308 users (Fig. 14) from all over the Arab world (Fig. 15).

Regarding the capacity building issue, many training sessions were organized with collaboration of national committees in Arab countries. There were 07 face-to- face training workshops so far using e-learning facilities to enhance the quality of training. These workshops were held in Tunisia, Doha, UAE, and Oman (Fig. 16). Some 150 participants from different majors, basically students and teachers, attended these sessions. Besides the training programme $\mathrm{M}$ Developer, described above in section 4, with more than 500 beneficiaries, all students from Tunisian universities. ALECSO plans to duplicate this program in other Arab countries with collaboration of local ministries of education and ICT. Another MOOC on mobile development entirely in Arabic language is still under preparation and will be launched shortly for all interested people from Arab countries.

\section{Conclusion}

The last recent years have witnessed tremendous growth of ICT and increasing availability of high 


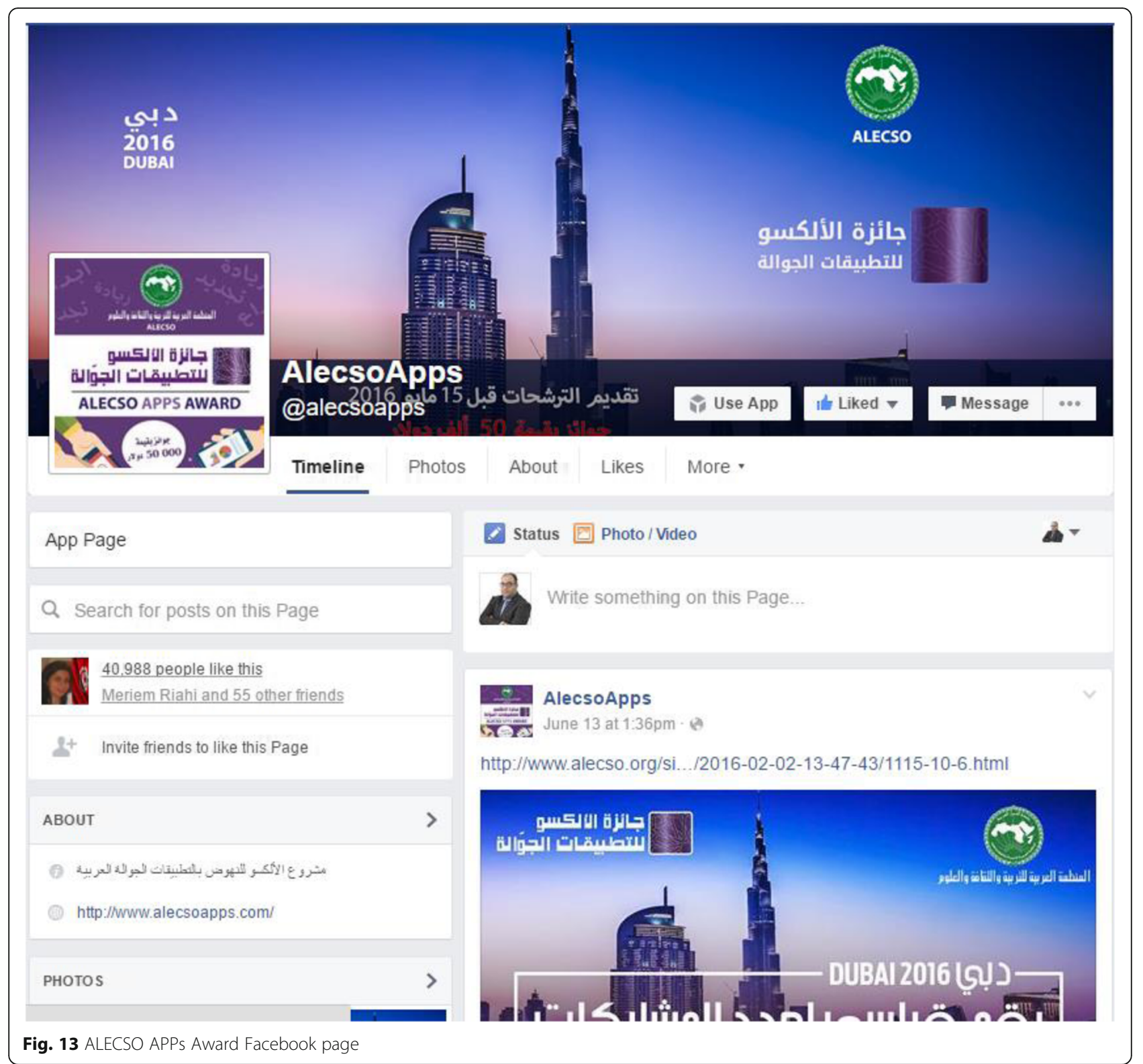

bandwidth mobile network infrastructures, which has opened up new opportunities for all sectors at all levels. Mindful of these major advancements in mobile technologies and big shifts in digital economy, ALECSO Organization committed to prepare and boost the ecosystem in the region in order to advance opportunities further for citizens especially in the education, culture and science fields. To this end, a comprehensive mobile initiative has been formulated and implemented by ALECSO since 2015, with special focus on building capacities aspects in order to empowering new generations of Arab developers with all required skills and competencies to innovate, create and develop Arab mobile applications and push forward towards Arab knowledge societies. In this paper, we presented the ALECSO Mobile initiative aiming to promote the mobile applications development and use in the Arab region. We put the focus on all its related projects and activities, especially the ALECSO Apps Store, Editor, Award, and training programs.

\section{Endnotes}

${ }^{1}$ https://itunews.itu.int/En/6266-The-State-of-Broadband-2015-.note.aspx

${ }^{2}$ http://www.gsma.com/ 




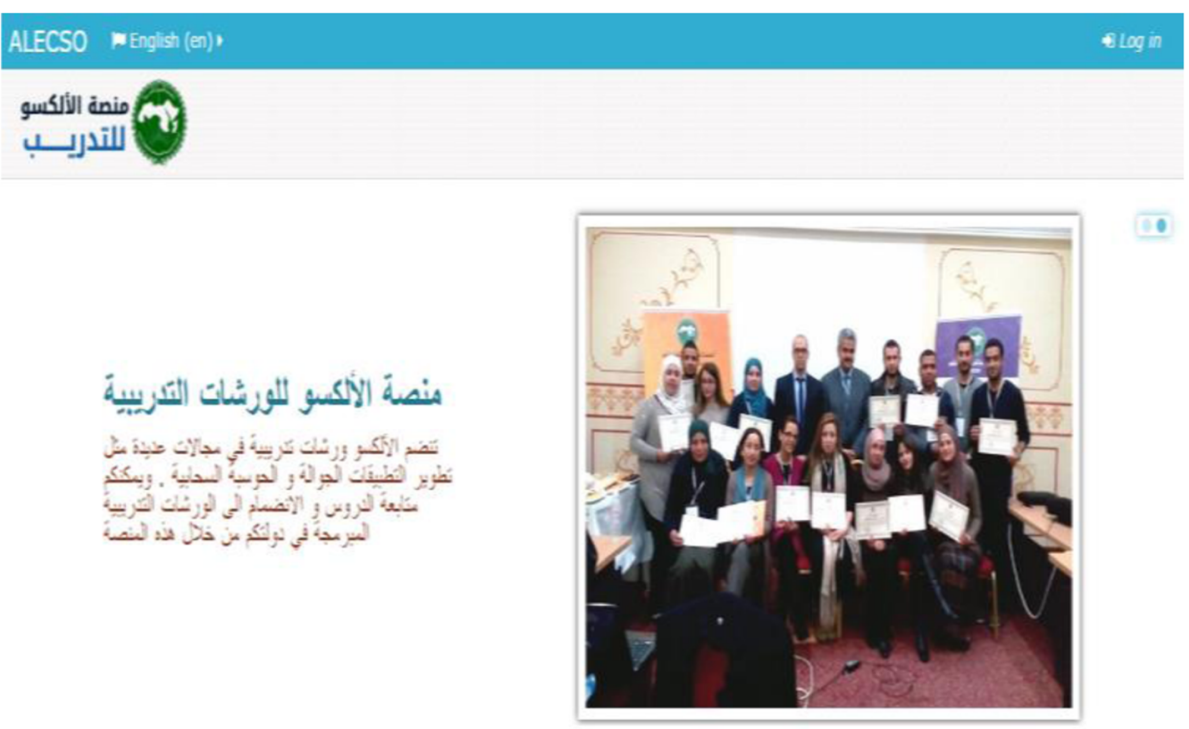

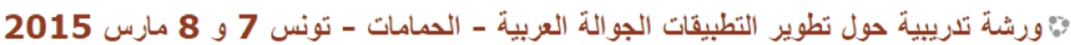
$[\rho$

Heithem Abbes :

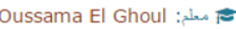
Ramzi FARHAT : Mohamed koutheair khribi :

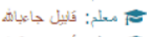

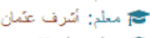

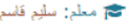

جوْْوة تلريبية حول تطوير التطبيقات الجوالة العربية - الاوحة - قطر - 10 و 11 و 12 مارس

2015

$[8$

Heithem Abbes : معلم:

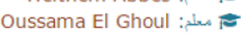
Ramzi FARHAT : Mohamed koutheair khribi : مطل

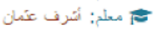

وفجورشة تلريبية حول تطوير النطبيقات الجوالة العربية - مسقط - سلطنة عمان -25 و26 و27

أكتوبر 2015

Fig. 16 ALECSO training workshops on Mobile Development available online on the ALECSO training platform

${ }^{3}$ http://en.unesco.org/youthmobile ${ }^{4}$ https://www.imaginecup.com ${ }^{5}$ http://www.arabmobilechallenge.com/

${ }^{6}$ http://www.alecsoapps.com

${ }^{7}$ http://http://www.alecsoapps.com

${ }^{8}$ http://www.android.com

${ }^{9}$ http://www.phonegap.com

${ }^{10} \mathrm{http}: / /$ training.alecso.org/

\section{Acknowledgements}

We would like to thank Prof. Abdullah Hamad Muharib, the Director General of the Arab League Educational, Cultural and Scientific Organization (ALECSO), who provided insight, wisdom and engagement that greatly helped in achieving ALECSO ICT projects to come to fruition. We also thank Mr. Oussema Elghoul, Mr. Achraf Othmen, Mr. Kabil Jaballah, the team of experts that worked on the ALECSO mobile initiative.

\section{Authors' contributions}

Both authors read and approved the final manuscript.

\section{Publisher's Note}

Springer Nature remains neutral with regard to jurisdictional claims in published maps and institutional affiliations.

Received: 2 January 2017 Accepted: 5 March 2017

Published online: 28 March 2017

\section{References}

GSM Association. The mobile economy. 2015a.

Association GSM and ATKEARNEY. The mobile economy 2013. 2013.

GSM Association. The mobile economy - arab states 2015. 2015b.

G. Business. Taping the App market. 2015.

Jemni M. Promoting the Effective Use of ICT for Enhancing Education in the Arab World. In: The 14th IEEE International Conference on Advanced Learning 
Technologies - ICALT2014 Advanced Technologies for Supporting Open Access to Formal and Informal Learning. 2014.

ALECSO and ITU. Guidelines for formulating national strategies on smart learning. 2015.

Butcher N. A basic guide to open educational resources OER, Commonwealt of learning and UNESCO. 2011.

The United Nations Educational Scientific and Cultural Organization UNESCO. TURNING ON MOBILE LEARNING : illustrative initiatives and policy implications. 2012a.

Kukulska-Hulme A, Sharples M, Milrad M, Arnedillo-Sanchez I, Vavoula G. The genesis and development of mobile learning in Europe. In: Hershey $P$, Parsons D, editors. Combining E-learning and M-learning: New applications of blended educational resources. 2011.

GSMA Intelligence. The mobile economy - Asia pacific 2015. 2015.

The United Nations Educational Scientific and Cultural Organization UNESCO. TURNING ON MOBILE LEARNING in Asia : illustrative initiatives and policy implications. 2012b.

The United Nations Educational Scientific and Cultural Organization UNESCO. TURNING ON MOBILE LEARNING in north america: illustrative initiatives and policy implications. 2012c

Jemni M, Khribi K, Achraf O, Oussama E, Kabil J. ALECSOApps: Toward Empowering Mobile Applications Development in the Arab World. In: International Conference on Smart Learning Environments (ICSLE 2015). p. $87-93$

Quillen I. Districts tackle questions regarding BYOT policy. 2011. [Online]. Available: http://www.edweek.org/dd/articles/2011/10/19/01 byot.h05.html.

Holzer A, Ondrus J. Mobile application market: A developer's perspective. 28. Netherlands: Telemat. Informatics, Elsevier B.V; 2011. p. 22-31.

Shotts K. PhoneGap 2.x mobile application development hotshot. 2013.

James M. Rapid application development. 1991

Jemni M, Khribi MK. The ALECSO Smart Learning Framework. In: Proceedings of International Conference on Smart Learning Environments (ICSLE 2016). p. 28-30.

Jemni M, Khribi MK. Toward empowering Open and Online Education in the Arab world through OER and MOOCs". In: Jemni M, Kinshuk, Khribi MK, editors. Open Education: from OERs to MOOCs, Springer Lecture Notes in Educational Technology series. 2016.

\section{Submit your manuscript to a SpringerOpen ${ }^{\circ}$ journal and benefit from:}

- Convenient online submission

- Rigorous peer review

- Immediate publication on acceptance

- Open access: articles freely available online

- High visibility within the field

Retaining the copyright to your article 